\title{
Re-expansion pulmonary edema after large left pleural effusion
}

\begin{abstract}
Background: Reexpansion pulmonary edema (REPE) is a complication that arises from a precipitous or rapid expansion of a collapsed lung. This rare complication is thought to arise after using an intercostal drainage tube in patients with a tension pneumothorax, those with large pleural effusions, and occasionally when used therapeutically in thoracentesis. There are a multitude of risk factors, and it usually self-limiting, with the mainstay of treatment being supportive with oxygen. It is believed that $20 \%$ of cases are fatal.

Case report: A 60-year-old male was treated with a 28 French tube for a large left pleural effusion. He developed REPE shortly after and developed severe shortness of breath, for which he required high flow oxygen. He was managed in the Intermediate Care Unit (IMCU) and shortly after was discharged home.
\end{abstract}

Keywords: reexpansion pulmonary edema, pneumothorax, pleural effusion, pulmonary edema, shortness of breath
Volume 8 Issue I - 202 |

\author{
Bilal Chaudhry, MD,' Kirill Alekseyev, MD, \\ MBA, ${ }^{2}$ Lidiya Didenko MS4, ${ }^{2}$ Nikita Donti, \\ DO' \\ 'Christiana Care Health System, USA \\ 2Post-Acute Medical Rehabilitation Hospital of Dover, American \\ University of Antigua, USA
}

Correspondence: Lidiya Didenko, Post-Acute Medical
Rehabilitation Hospital of Dover. I 240 McKee Road, Dover, DE
19904, USA, Tel (862)377-1907, Email lidiyadid@yahoo.com

Received: February 22, 2021 | Published: March 09, 2021
Abbreviations: REPE, reexpansion pulmonary edema; IMCU, intermediate care unit; ED, emergency department; SOB, shortness of breath; PCP, primary care physician; PERRL, pupils were equal, round, reactive to light; EOMI, extraocular movements were intact; 28 F, 28 French chest tube; CPAP, continuous positive airway pressure; BiPAP, bilevel positive airway pressure

\section{Introduction}

Reexpansion pulmonary edema (REPE) is a rare but serious complication that occurs in about $1 \%$ of the time following the rapid expansion of a collapsed lung. Initially the patients may experience a sudden onset cough in addition to shortness of breath (SOB). There are multiple risk factors in the development of REPE. Some of the leading risk factors include young age, large pneumothoraxes, or a persistent pneumothorax for more than 3 to 7 days and lastly, those that drain more around 3 or more liters. REPE can be diagnosed in the clinical setting with a CT or x-ray. Some of the most notable x-ray findings include edema of the affected lung and alveolar opacities. Some of the classical findings in CT include ground glass opacification of the affected lung, in addition to smooth interstitial thickening. The mainstay of treatment is supportive with oxygen supplementation via ventilatory, or other non-invasive modalities. Additionally, morbidity and mortality can be reduced in these patients if the are placed in a lateral decumbency position with the affected lung superior.

\section{Case report}

We present a 60 -year-old male that presented to the emergency department (ED) with a complaint of shortness of breath (SOB) associated with a cough. Prior to this, he had a nonproductive cough for two weeks, which prompted the patient to visit his primary care physician (PCP). He was then prescribed antibiotics. He did not see any improvement, and after a few days, he developed SOB and decided to return to his PCP.

He was then sent for a chest $\mathrm{x}$-ray, which demonstrated that he had left lung atelectasis with effusion. At that point, he was recommended to go to the ED. He denied any sick contacts, fevers, chills, nausea, vomiting, weight loss, night sweats, heart palpitations, diarrhea, constipation, any recent travel, any personal history of tuberculosis, or any tuberculosis contact. He denied the use of drugs and was not a smoker. He further denied any family history of cancer, lung disease, or any rheumatologic diseases as well.

Table I Pleural fluid analysis: Exudative pleural effusion. Additionally, the pleural fluid was negative for malignancy

\begin{tabular}{ll}
\hline Pleural Fluid & Values \\
\hline $\mathrm{pH}$ & 7.5 \\
Albumin & $2.3 \mathrm{~g} / \mathrm{dL}$ \\
Amylase & $27 \mathrm{U} / \mathrm{L}$ \\
Glucose & $88 \mathrm{mg} / \mathrm{dL}$ \\
$\mathrm{LDH}$ & $439 \mathrm{U} / \mathrm{L}$ \\
WBC & $1283 / \mathrm{mm}^{3}$ \\
Organisms & Negative \\
Malignant cells & Negative
\end{tabular}

In the ED, he received another chest x-ray, which showed a left pleural effusion. A review of systems revealed a skinny, alert and cooperative male that appeared to be in no acute distress. His head was normocephalic; pupils were equal, round, reactive to light (PERRL), extraocular movements were intact (EOMI). Upon cardiac auscultation, there was a normal S1, S2, and no S3, S4, murmurs, rubs, or gallops. The rhythm was regular, and there was no peripheral edema, cyanosis or pallor. Auscultation of the lungs revealed diminished breath sounds on the left. The abdomen was positive for bowel sounds, it was soft, non-distended, non-tender, and there was no guarding, rebound, or masses. There was no significant deformity or joint abnormality, peripheral pulses were intact, and there were no varicosities. Skin appeared normal color, texture, and turgor with no lesions or eruptions. Cranial nerves II-XII were intact. He had normal affect and behavior.

The patient then had a CT of the chest that showed a large left pleural effusion resulting in complete atelectasis of the left lung in addition to trace right pleural effusion. Cardiothoracic surgery was then consulted, and the decision to place a chest tube was made. 
He received a left 28 French (28F) chest tube with a water seal. He proceeded to drain about 3.4L throughout the day. The patient felt that his breathing was improving at this point.

A chest $\mathrm{x}$-ray was ordered once he drained about $2 \mathrm{~L}$, and his new imaging showed a decrease in the size of the pleural effusion with slight increased aeration of the left upper lobe. The left chest tube was in place and terminated near the left suprahilar region. The cardiac silhouette was partially obscured, and the right lung remained clear. Additionally, a fluid analysis and culture was ordered and revealed an exudative effusion that was negative for cultures. This was followed up with fluid studies and cytology. Figure 1 depicts the findings from the pleural fluid analysis. There were many inflammatory cells with scattered reactive mesothelial cells and macrophages.

Given his history suggestive of an infectious process, likely pneumonia, which caused the pleural effusion, a Pulmonary and Infectious Disease consult was ordered. He was started once again on his antibiotics, which consisted of ceftriaxone and doxycycline.

\section{Discussion}

Reexpansion pulmonary edema occurs because of the expansion of a collapsed lung. The symptomatology can vary widely. In a mild case, the patient may be asymptomatic, and the REPE is only visualized on a radiograph post-treatment. In a moderate case, symptomatology can include SOB and a dry cough. While in more severe cases, the patient may experience severe cardiopulmonary collapse with hypotension and tachycardia. ${ }^{1}$ Additionally, nearly $20 \%$ of cases result in death. ${ }^{2,3}$

The pathogenesis behind REPE is not well known. Mahfood et al. stated back in 1988 that the chronicity of pulmonary collapse, airway obstructions, increased permeability of the pulmonary vasculature, pulmonary artery pressure changes, and loss of surfactant can all play a role in the development of REPE. ${ }^{3}$ Additionally, it is believed that the removal of more than $2000 \mathrm{ml}$ at once, using negative pressure, and the reexpansion of the lung in less than 10 minutes can also lead to the development of REPE. ${ }^{4,5}$ There are also certain risk factors that play a role in the development of REPE, and they include female sex and younger age.

Rozenman et al. notes that polymorphonuclear leukocyte (PMN) accumulation, various fluid mediators, and protein leakage has been noted in the pleura fluid in these patients. These fluid mediators are believed to be involved in the chemotaxis and activation of the PMN that leads to the development of the edema seen in REPE.

The degree of the edema typically correlates to the signs and symptoms that the patient develops, and these signs and symptoms can vary in a patient with REPE. The typical symptoms in patients with the development of edema is a cough and dyspnea. ${ }^{2}$ Patients that develop symptoms usually develop them within a couple of minutes up to 48 hours since the procedure was done. Additionally, a CT in these patients will reveal ground-glass opacities, areas of atelectasis, focal consolidation, and possibly septal thickening. ${ }^{4}$

When considering REPE, the differential diagnosis for pneumonia or unilateral cardiogenic pulmonary edema should be kept in mind, especially if it is unilateral presentation of chest radiographs. A normal WBC count, absence of fever, and normal cardiovascular exam would help rule out pneumonia and unilateral cardiogenic pulmonary edema. ${ }^{1}$ When REPE diagnosis is established, prompt treatment can then be initiated. The main goal of treatment is to provide the patient with sufficient oxygenation, whether it be additional oxygen via in a nasal cannula, Continuous positive airway pressure (CPAP), Bilevel positive airway pressure (BiPAP), and in extreme cases, full mechanical ventilatory support until the pulmonary edema resolves.

\section{Conclusion}

Although relatively uncommon, REPE is a potentially fatal and rare complication that arises from precipitous or rapid expansion of the lung that is collapsed. This can lead to respiratory failure if not caught promptly. Early identification and management of REPE increases the chances of a favorable outcome. Initial warning signs and symptoms include a cough, dyspnea, and low oxygen saturation after the drainage or lung reexpansion. Supportive therapy via oxygen supplementation will ultimately lead to resolution. It is imperative that physicians catch and treat REPE early in its course due to the lethality of further complications.

\section{Acknowledgments}

None.

\section{Conflicts of interest}

None.

\section{References}

1. Rozenman J, Yellin A, Simansky DA, et al. Re-expansion pulmonary oedema following spontaneous pneumothorax. Respir Med. 1996;90(4):235-238.

2. Verhagen M, van Buijtenen JM, Geeraedts LM. Reexpansion pulmonary edema after chest drainage for pneumothorax: A case report and literature overview. Respir Med Case Rep. 2015;14:10-12.

3. Mahfood S, Hix WR, Aaron BL, et al. Reexpansion pulmonary edema. Ann Thorac Surg. 1988;45(3):340-345.

4. Avinash RM, Poovil AA, Nagendra D, et al. Reexpansion pulmonary edema in a young lady post- intercostal drainage tube insertion. $J$ Med Sci Res. 2018; 6(3):93-95.

5. Sarda S, Verma A, Jaiswal S, et al. Reexpansion pulmonary edema: A rare complication of pneumothorax drainage. Turk $J$ Emerg Med. 2020;20(4):196-198. 